
\title{
Research S Suare \\ Dual Band Thin Film Infrared Detector Design and Performance Improvement using Plasmonic Nanostructure
}

Mohammad Bashirpour ( $\sim$ m.bashirpour@urmia.ac.ir)

Urmia University https://orcid.org/0000-0002-7622-564X

Saeed Khankalantary

Isfahan University of Technology

Mohammadreza Kolahdouz

University of Tehran

\section{Research Article}

Keywords: Infrared, Dual band photodetector, Plasmonic, Nanostructure, Thin film.

Posted Date: August 13th, 2021

DOI: https://doi.org/10.21203/rs.3.rs-774208/v1

License: (c) (i) This work is licensed under a Creative Commons Attribution 4.0 International License.

Read Full License 


\title{
Dual Band Thin Film Infrared Detector Design and Performance Improvement using Plasmonic Nanostructure
}

\author{
Mohammad Bashirpour ${ }^{1, *}$, Saeed Khankalantary ${ }^{2}$, Mohammadreza Kolahdouz ${ }^{3}$ \\ ${ }^{1}$ Department of Electrical and Computer Engineering, Urmia University, Urmia, Iran \\ ${ }^{2}$ Department of Electrical and Computer Engineering, Isfahan University of Technology, \\ Isfahan, Iran \\ ${ }^{3}$ School of Electrical and Computer Engineering, College of Engineering, University of Tehran, \\ Tehran, Iran \\ *Corresponding author: m.bashirpour@urmia.ac.ir
}

\begin{abstract}
A new dual band thin film metal-semiconductor-metal infrared photodetector base on InGaAs for wavelength of 1.1-1.7 $\mu \mathrm{m}$ and InSb for wavelength of 3-5 $\mu \mathrm{m}$ is proposed and investigated numerically. One major problem of thin film photodetectors is low quantum efficiency that originates from low optical absorption. The quantum efficiency of proposed structure is improved by locating the array of optimized aluminum nanostructure (Al-NS) between the InGaAs and InSb layers. Using optimized Al-NS between the stack of InGaAs and InSb (InSb/Al-NS/InGaAs) results in plasmon excitation inside the photosensitive layers and so, higher photocarrier generation. Moreover, locating zinc oxide nanorode as an antireflection coating on top of detector reduces the incident light reflection in both spectrum of 1.1-1.7 $\mu \mathrm{m}$ and 3-5 $\mu \mathrm{m}$. The finite different time domain method is used to investigated the optical properties of proposed structure and optimize the structure. According to the simulation results, designed structure gives rise to $108.1 \%, 110 \%$ and $320 \%$ light absorption enhancement at wavelength of $1.33 \mu \mathrm{m}, 1.55 \mu \mathrm{m}$ and $4 \mu \mathrm{m}$, respectively compared to reference conventional structure.
\end{abstract}

Keywords: Infrared; Dual band photodetector; Plasmonic; Nanostructure; Thin film. 


\section{Introduction}

Recently, dual band infrared (IR) photodetectors has attracted lots of attention due to its ability to detect two different wavelengths band separately and independently [1]. Dual band detection at near infrared (NIR) and middle wave infrared (MWIR) have lots of application, including environmental monitoring, night vision devices, infrared spectroscopy, target tracking and, etc. This kind of IR detectors benefit from better image contrast, higher resolution and longer detection distant compared to conventional single band detectors [2]. Conventional IR detectors are fabricated on photosensitive material such as InGaAs, InSb, $\mathrm{HgCdTe}, \mathrm{InP}, \mathrm{InAs}, \mathrm{PbI}_{2}$ and etc [3-8], with large thickness that can cause some difficulties such as high production cost and large stress. Using thin film as a photosensitive layer reduces the production cost and complexity of high quality material deposition process and leads to decrease in dark current of detector [9]. Moreover, thin film detectors can be fabricated on flexible substrates that have attracted lots of attention in the last decade due to the new area of researches. The major drawback of thin film detector is its low optical absorption due to the short optical path inside the photosensitive material. Therefore, physical investigation of optical absorption mechanism inside the thin film IR photodetector and finding a scheme to enhance the optical absorption is critical for its application at imaging and detection [10]. Lots of research is done to improve the performance of IR photodetectors by increasing the optical absorption, such as, graphene plasmon excitation [11,12], plasmonic enhanced carbon nanotube detector [13], muli-layer hetro-structure [14,15], Fabry Perot cavity resonance[16], dielectric photonic crystals [17], distributed brag reflectors [18], and surface plasmon excitation $[19,20]$. Localized surface plasmon resonance (LSPR) in the interface of noble metals leads to the strong local field and hot points inside the photosensitive layers and makes this technology an effective method for light-matter interaction at subwavelength dimensions. Strong local field results in more photocarrier generation and so the enhancement of photocurrent [21-23]. Thanks to the new nanostructure fabrication methods, different kind of metallic nanostructure have been applied to the wide range of optoelectronic devices to enhance the light-matter interaction, such as, solar cell, photoconductive source and detectors, photodetectors, and etc $[24,25]$. Most of the LSPR research work is done at visible rang of electromagnetic 
spectrum that indicates the vital need to study this area of science at IR spectrum. Multiband photodetector as a next generation of IR detectors, can benefit from multi band resonance of plasmonic structure due to the multiple resonance mode of single array of plasmonic nanostructure $[26,27]$.

Dual band thin film IR detector at NIR and MWIR band based on InGaAs and InSb thin film is proposed in this work. An optimized array of aluminum plasmonic nanostructure is applied in order to increase the optical absorption of incident light inside the photosensitive layers by excitation of surface plasmon. Aluminum nanoparticles (Al-NP) have wide spectral range of absorption including IR. By designing the geometrical parameters and locating the Al-NPs array between the stack of InGaAs and InSb layers, the optical path of light inside the photosensitive layers increases and results in higher optical absorption.

\section{Structure design and simulation method}

The configuration of proposed thin film dual-band IR detector is illustrated in Fig. 1. Proposed structure consist of a layer of InSb with thickness of $2 \mu \mathrm{m}$ located on sapphire substrate. An array of aluminum nanostructure (Al-NS) is placed on InSb layer. It can be fabricated by electron beam lithography followed by sputtering and lift-off process. Layer of $\mathrm{SiO}_{2}$ with thickness of $100 \mathrm{~nm}$ deposited on $\mathrm{Al}-\mathrm{NS}$ and $500 \mathrm{~nm} \mathrm{InGaAs}$ put on $\mathrm{SiO}_{2}$ layer. At the end, $\mathrm{ZnO}$ nanorode as an antireflection coating and light concentrator located on top of the proposed device. The periodicity and diameter of Al-NS are optimized to achieve the maximum absorption inside the photosensitive layer. The electrodes are located on InSb layer and contacted to InGaAs layer to collect both layers photocarriers. 


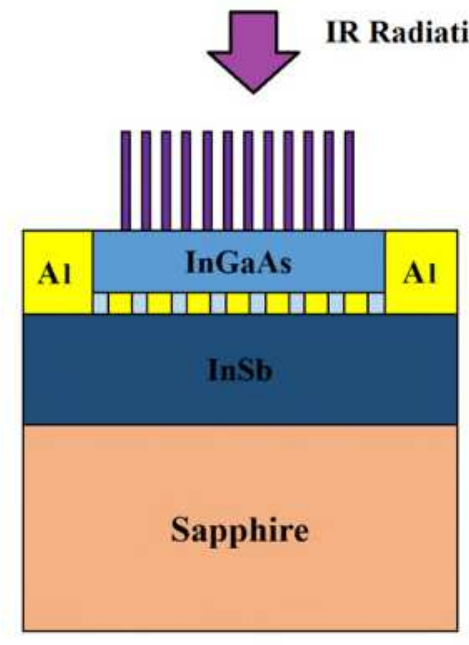

(a)

\begin{tabular}{|c|c|c|}
\hline Al & ARC & Al \\
\hline \multicolumn{3}{|c|}{ InGaAs } \\
\hline
\end{tabular}

(b)

\begin{tabular}{|c|c|c|}
\hline Al & ARC & Al \\
\hline \multicolumn{3}{|c|}{ InSb } \\
\hline
\end{tabular}

(c)

Fig. 1. Schematic diagram of a) proposed thin film dual band metal-semiconductor-metal IR detector (structure A) b) reference conventional InGaAs based IR detector (structure B) c) reference InSb based IR detector (structure C).

Finite difference time domain method (FDTD) is applied to solve the Maxwell's wave equations and investigate the optical properties of proposed structure. This numerical method have been used to evaluate the reflection and absorption spectra of incident light from the surface and inside the photosensitive layers. The electric field distribution inside the layers is calculated in order to investigate the local field enhancement using plasmonic structure. The perfectly matched layer (PML) is used at boundaries of simulation region. To obtain promising results, the material's refractive index are extracted from previously published experimental data [28].

\section{Results and discussion}

The interaction of light quasi particle (photon) with oscillating electrons at the interface of metal-dielectric is called surface plasmon polariton (SPP) that causes high local field in the deep sub wavelength dimension. There is two kind of SPPs: localized SPP and propagating SPP. The excited surface plasmon wave can propagate along the interface as far as several micrometers. In order to excite the propagating SPPs, the energy and momentum conservation between the incident light and plasmonic nanostructure has to be satisfied $[29,30]$. 
$k_{S P P}=k_{0} \sqrt{\frac{\varepsilon_{m} \varepsilon_{d}}{\varepsilon_{m}+\varepsilon_{d}}}$

Where $k_{0}$ is incident light wavevector, $\varepsilon_{m}$ is metal permittivity, $\varepsilon_{d}$ is dielectric permittivity, and $k_{S P P}$ is SPPs wavevector. As can be seen in Eq. 4, a nanograting coupling structure has to be used in order to match the incident light wavevector to SPPs wavevector.

$k_{S P P}=k_{i n} \sin \theta \pm m k_{g}$

$k_{g}=\frac{2 \pi}{\lambda}$

$p=\alpha \lambda\left(\sqrt{\frac{\varepsilon_{m} \varepsilon_{d}}{\varepsilon_{m}+\varepsilon_{d}}}\right)^{-1}$

Where, the wavevector of plasmonic nanograting is $k_{g}, \theta$ is the incident light angle with surface, and $p$ is the periodicity of plasmonic nanograting. In this work, the plasmonic structure has been designed carefully to excite the SPPs in both NIR and MWIR range. The designed nanostructure located between the layer of InGas and InSb which results to high local field inside the photosensitive layers at the wavelength of 1.1-1.7 $\mu \mathrm{m}$ and $3-$ $5 \mu \mathrm{m}$. Electric field distribution inside the InGaAs layer for conventional structure (Structure B) and proposed IR detector with aluminum nanoparticles array with periodicity (P) of $440 \mathrm{~nm}$, diameter (D) of $200 \mathrm{~nm}$ and height $(\mathrm{H})$ of 100 (Structure A) is shown at Fig. 2b. As can be seen, the Al-NS under the InGaAs excite the SPP and results in high local field inside the InGaAs layer. According to the simulation results, electric field shows more than 6 times enhancement compared to conventional structure. Higher electric field results in more photocarrier generation and, so the photodetector photocurrent enhancement. 


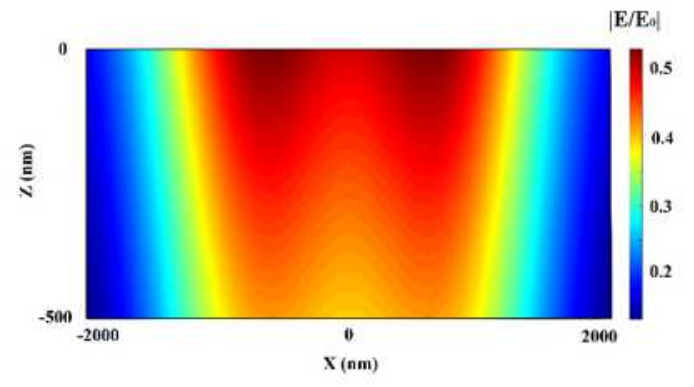

(a)

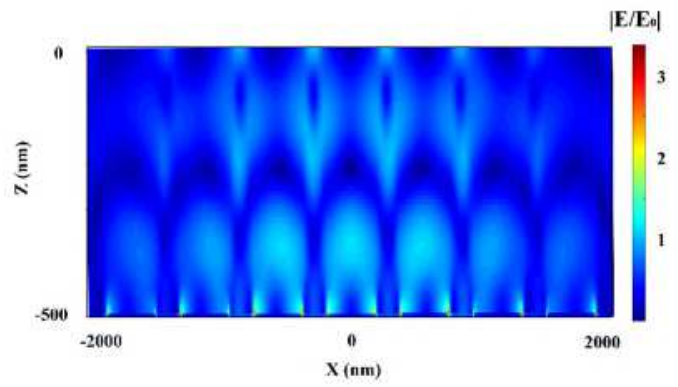

(b)

Fig. 2. Electric field distribution of a) conventional InGaAs structure (Structure B) b) Proposed structure at nanoparticle array periodicity of $450 \mathrm{~nm}$, diameter of $225 \mathrm{~nm}$ and height of 100

In order to achieve the maximum absorption inside the InGaAs layer, the geometrical parameters of designed Al-NS is optimized. In the first step, the height and diameter of Al-NS set to $100 \mathrm{~nm}$ and $200 \mathrm{~nm}$, respectively and the periodicity swept from $300 \mathrm{~nm}$ to $600 \mathrm{~nm}$ with $10 \mathrm{~nm}$ steps. As can be seen in Fig. 3a, there is two absorption peak that are related to SPP excitation. The peak of absorption moves to higher wavelength by increasing the periodicity of Al-NS. The highest absorption of $74.8 \%$ at wavelength of $1.33 \mu \mathrm{m}$ and $63.5 \%$ at wavelength of $1.55 \mu \mathrm{m}$ (two important wavelength for IR imaging and IR communication) obtained at periodicity of $440 \mathrm{~nm}$ (Fig. 3a). In the next step, the periodicity set to $440 \mathrm{~nm}$ and the Al-NS diameter swept from $100 \mathrm{~nm}$ to $300 \mathrm{~nm}$ with 10 nm steps. The absorption of $74.9 \%$ at wavelength of $1.33 \mu \mathrm{m}$ and $63.7 \%$ at wavelength of $1.55 \mu \mathrm{m}$ achieved at diameter of $210 \mathrm{~nm}$.

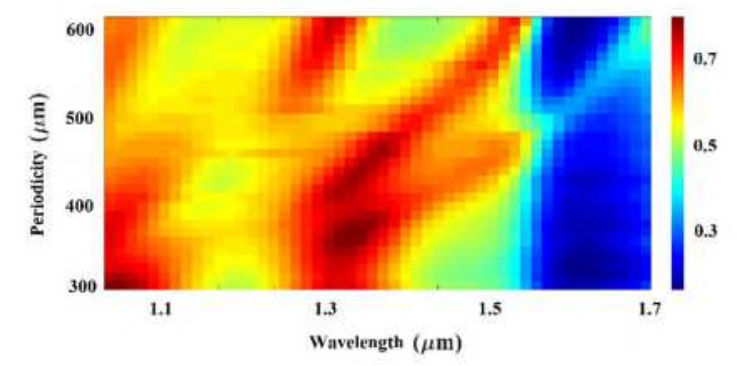

(a)

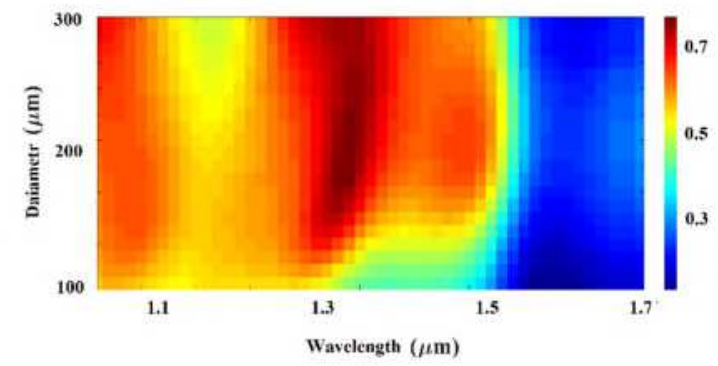

(b)

Fig. 3. a) Absorption as a function of wavelength and periodicity for $\mathrm{D}=200 \mathrm{~nm}$ and $\mathrm{H}=100 \mathrm{~nm}$ b) Absorption as a function of wavelength and Diameter for $\mathrm{P}=440 \mathrm{~nm}$ and $\mathrm{H}=100 \mathrm{~nm}$ 
Fig. $4 \mathrm{a}$ and $4 \mathrm{~b}$ show the absorption and reflection of optimized structure (structure A) as a function of wavelength inside the InGaAs layer compared to conventional metalsemiconductor-metal (MSM) detector (structure B). As can be seen in Fig. 4a, the absorption shows more than $108 \%$ enhancement at wavelength of $1.33 \mu \mathrm{m}$ and $110 \%$ enhancement at wavelength of $1.55 \mu \mathrm{m}$ compared to structure B. The Al-NSs under the InGaAs layer excite the SPP and result in high local field inside the layer. Higher local field leads to more photocarrier generation and so the enhancement of photocurrent. Moreover, Al-NSs under the photosensitive layer reflect back the transmitted light from the bottom in to the InGaAs layer and enhance the optical path of incident light. This phenomena results in more photocarrier generation and so the enhancement of photocarrier. The zinc oxide nanorode ( $\mathrm{ZnO}-\mathrm{NR})$ height fixed to $1.2 \mu \mathrm{m}$ in order to obtain highest absorption at 1.1-1.7 $\mu \mathrm{m}$. The reflection of 1.1-1.7 $\mu \mathrm{m}$ wave from the surface of detector is illustrated for structure A, structure B, and structure B without anti reflection coating in Fig. 4b. Reflection between 1.3-1.5 $\mu \mathrm{m}$ has reduced to less than $5 \%$ for proposed structure (structure A). Zinc oxide nanorode on top of the InGaAs layer acts as an antireflection coating and light concentrator which helps to enhance the IR wave absorption inside the photosensitive layer.

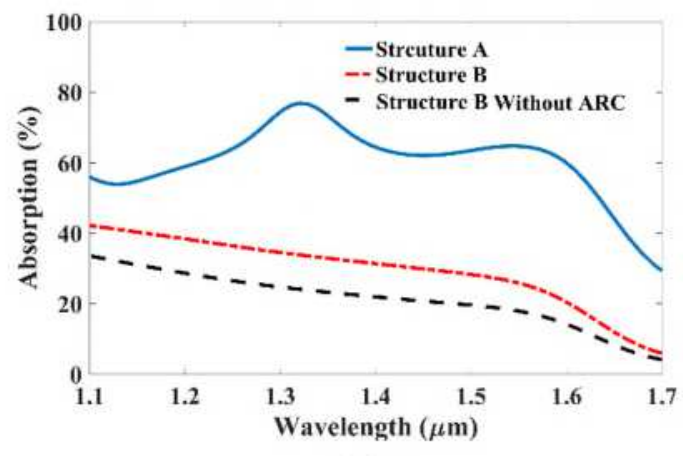

(a)

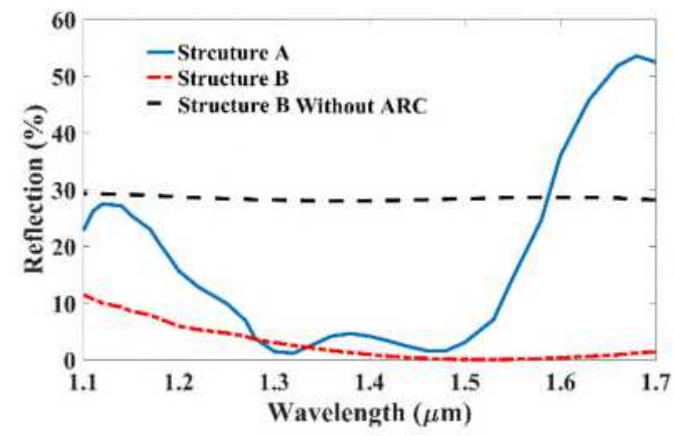

(b)

Fig. 4. Diagram of absorption as a function of wavelength for proposed structure (structure A), structure B, and structure B without ARC b) diagram of reflection as a function of wavelength for proposed structure (structure A), structure B, and structure B without ARC

The IR detector based on InGaAs can only detect the incident photons with smaller wavelength than its energy gap. In order to detect the wavelength of 3-5 $\mu \mathrm{m}$, the InSb with thickness of $1 \mu \mathrm{m}$ on sapphire substrate located under the Al-NS. The height of 
nanorode can be optimized to achieve higher absorption inside the InSb layer. The absorption inside the InSb layer as a function of wavelength for proposed structure (structure A), structure A without $\mathrm{ZnO}$ NR and structure $\mathrm{C}$ as a reference is illustrated in Fig.5. The $\mathrm{ZnO} \mathrm{NRs}$ act as a graded refractive index antireflection coating. Moreover, ZnO NRs leads to higher optical absorption by concentrating the incident IR light. As can be seen in Fig. 5, the absorption inside the InSb layer for proposed structure shows more than $76 \%$ enhancement at wavelength of $4 \mu \mathrm{m}$ compared to structure A without $\mathrm{ZnO}$ NRs and $142 \%$ enhancement compared to structure C.

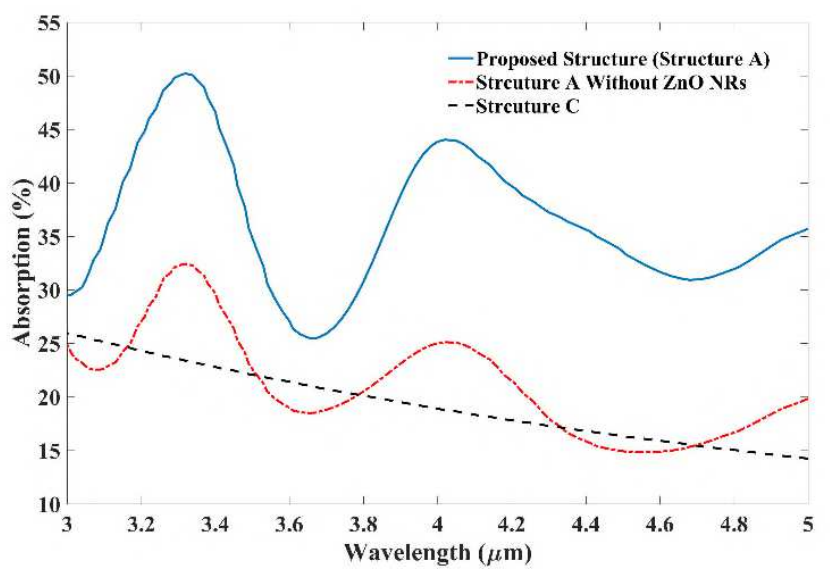

Fig. 5. Diagram of absorption as a function of wavelength for proposed structure (structure A), structure A without $\mathrm{ZnO}$ NRs and structure $\mathrm{C}$

The electric field distribution inside the InSb layer for proposed structure (structure A) and reference structure (structure C) is shown in Fig. 6a and 6b. Array of Al-NPs on the InSb layer results in high local field points with more than 6 times enhancement compared to conventional structure. The Al-NPs geometrical parameters have designed carefully to excite the plasmon polariton in both 1.1-1.7 $\mu \mathrm{m}$ and 3-5 $\mu \mathrm{m}$ wavelength of IR spectrum. 


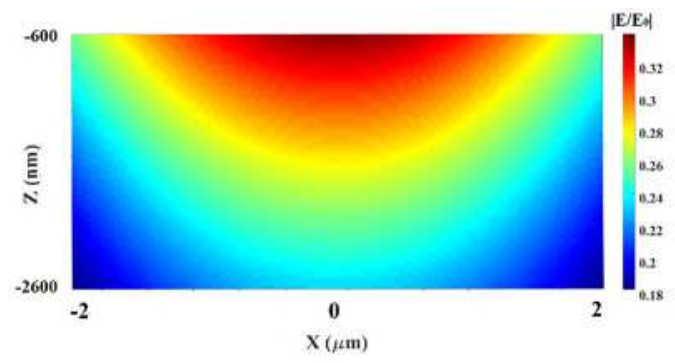

(a)

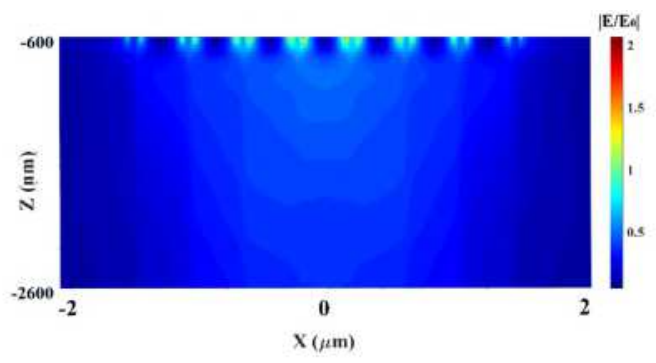

(b)

Fig. 6. Electric field distribution inside the InSb layer for a) structure C b) structure A

Finally, the whole proposed structure is simulated and the absorption and the electric field distribution are shown in Fig. 7a and 7b, respectively. The absorption of $73.8 \%, 63.8 \%$ and $84 \%$ at wavelength of $1.33 \mu \mathrm{m}, 1.55 \mu \mathrm{m}$ and $4 \mu \mathrm{m}$ is calculated that indicates the potential of proposed structure for multi-band IR detection at NIR, SWIR and LWIR bands. Zinc oxide NRs located on the IR detector surface instead of simple Si3N4 antireflection layer, results in low reflection at multi band incident wave. Moreover, $\mathrm{ZnO}$ NRs concentrate the incident wave inside the photo absorption layers and results in more photon absorption. As can be seen in Fig. 7b, using AL-NPs stacked between the InGaAs and InSb layer excites the SPPs and leads to high local field points inside the layers. This phenomena increases the photocarrier generation inside the photosensitive layers and results in higher photocurrent. Surface plasmon polariton has shorter wavelength than incident light. So, it is possible to use the plasmon wave to create photocarrier inside the InGaAs layer at MWIR rang.

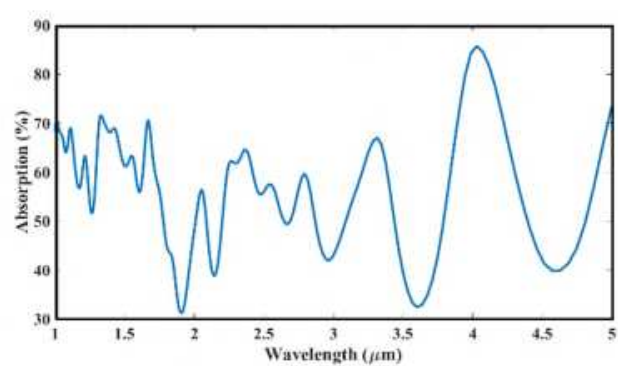

(a)

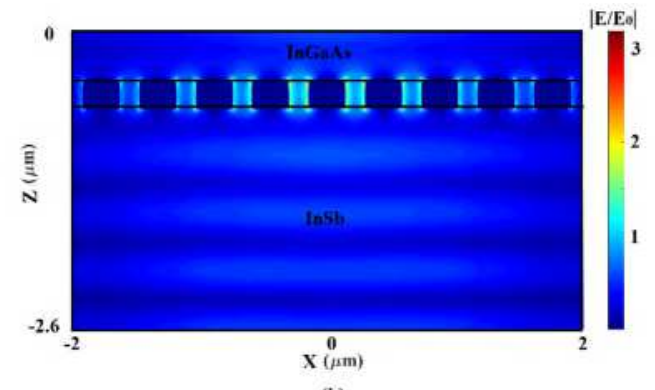

(b)

Fig. 7. Absorption as a function of wavelength for proposed structure at wavelength of $1-5 \mu \mathrm{m} b$ ) electric field distribution of proposed structure at wavelength of $4 \mu \mathrm{m}$ 


\section{Conclusion}

In summary, a new structure for multi band thin film IR detector at the NIR and MWIR is proposed and its performance investigated using finite difference time domain method. An array of AL-NPs is located between the InGaAs and InSb layer in order to excite the plasmonic wave inside the both photosensitive layers. According to the simulation results, by location the optimized Al-NPs between two photosensitive layers, the SPPs penetrate inside the layers and result in photocarrier generation enhancement and strong coupling of incident light to the photosensitive layer. The proposed structure gives rise to $108.1 \%$, $110 \%$ and $320 \%$ light absorption enhancement at wavelength of $1.33 \mu \mathrm{m}, 1.55 \mu \mathrm{m}$ and 4 $\mu \mathrm{m}$, respectively compared to reference conventional structure. These results pave the way for high performance thin film IR detector that can be used at multi band spectrum.

\section{Declaration}

The authors declare no conflict of interest. 


\section{Reference}

[1] J. Tong, L.Y.M. Tobing, Y. Luo, D. Zhang, D.H. Zhang, Single plasmonic structure enhanced dual-band room temperature infrared photodetection, Sci. Rep. 8 (2018) 1-9.

[2] A. Rogalski, HgCdTe infrared detector material: history, status and outlook, Reports Prog. Phys. 68 (2005) 2267.

[3] J. Hwang, Z. Ku, J. Jeon, Y. Kim, J.O. Kim, D.-K. Kim, A. Urbas, E.K. Kim, S.J. Lee, PlasmonicLayered InAs/InGaAs Quantum-Dots-in-a-Well Pixel Detector for Spectral-Shaping and Photocurrent Enhancement, Nanomaterials. 10 (2020) 1827.

[4] J. Sun, M. Han, Y. Gu, Z. Yang, H. Zeng, Recent Advances in Group III-V Nanowire Infrared Detectors, Adv. Opt. Mater. 6 (2018) 1800256.

[5] C.-C. Chang, Y.D. Sharma, Y.-S. Kim, J.A. Bur, R. V Shenoi, S. Krishna, D. Huang, S.-Y. Lin, A surface plasmon enhanced infrared photodetector based on InAs quantum dots, Nano Lett. 10 (2010) 1704-1709.

[6] C. Shi, Y. Dong, Q. Li, High-Performance Nonequilibrium InSb PIN Infrared Photodetectors, IEEE Trans. Electron Devices. 66 (2019) 1361-1367.

[7] S. Tyagi, P.K. Saxena, R. Kumar, Numerical simulation of InxGa1-xAs/InP PIN photodetector for optimum performance at 298 K, Opt. Quantum Electron. 52 (2020) 1-12. https://doi.org/10.1007/s11082-020-02488-1.

[8] R.A. Ismail, A.M. Mousa, S.S. Shaker, Visible-enhanced silver-doped PbI2 nanostructure/Si heterojunction photodetector: effect of doping concentration on photodetector parameters, Opt. Quantum Electron. 51 (2019) 1-19. https://doi.org/10.1007/s11082-019-2063-x.

[9] X. Luo, X. Zhai, L. Wang, Q. Lin, Enhanced dual-band absorption of molybdenum disulfide using a plasmonic perfect absorber, Opt. Express. 26 (2018) 11658-11666.

[10] C. Liang, Z. Yi, X. Chen, Y. Tang, Y. Yi, Z. Zhou, X. Wu, Z. Huang, Y. Yi, G. Zhang, Dual-band infrared perfect absorber based on a Ag-dielectric-Ag multilayer films with nanoring grooves arrays, Plasmonics. 15 (2020) 93-100.

[11] C. Guo, J. Zhang, W. Xu, K. Liu, X. Yuan, S. Qin, Z. Zhu, Graphene-based perfect absorption structures in the visible to terahertz band and their optoelectronics applications, Nanomaterials. 8 
(2018) 1033.

[12] Y. Zhang, D. Meng, X. Li, H. Yu, J. Lai, Z. Fan, C. Chen, Significantly enhanced infrared absorption of graphene photodetector under surface-plasmonic coupling and polariton interference, Opt. Express. 26 (2018) 30862-30872.

[13] H. Huang, F. Wang, Y. Liu, S. Wang, L.-M. Peng, Plasmonic enhanced performance of an infrared detector based on carbon nanotube films, ACS Appl. Mater. Interfaces. 9 (2017) 1274312749.

[14] M. Xiong, D. Su, H.-L. Zhou, J.-Y. Wu, S. Iqbal, X.-Y. Zhang, T. Zhang, Plasmonic enhanced mid-infrared InAs/GaSb superlattice photodetectors with the hybrid mode for wavelengthselective detection, AIP Adv. 9 (2019) 85121.

[15] M. Kopytko, W. Gawron, A. Kębłowski, D. Stępień, P. Martyniuk, K. Jóźwikowski, Numerical analysis of $\mathrm{HgCdTe}$ dual-band infrared detector, Opt. Quantum Electron. 51 (2019) 1-8.

[16] G. Kang, I. Vartiainen, B. Bai, J. Turunen, Enhanced dual-band infrared absorption in a FabryPerot cavity with subwavelength metallic grating, Opt. Express. 19 (2011) 770-778.

[17] J. Rosenberg, R. V Shenoi, S. Krishna, O. Painter, Design of plasmonic photonic crystal resonant cavities for polarization sensitive infrared photodetectors, Opt. Express. 18 (2010) 3672-3686.

[18] Y. Zhang, A. Haddadi, A. Dehzangi, R. Chevallier, M. Razeghi, Suppressing Spectral Crosstalk in Dual-Band Long-Wavelength Infrared Photodetectors With Monolithically Integrated Air-Gapped Distributed Bragg Reflectors, IEEE J. Quantum Electron. 55 (2018) 1-6.

[19] H. Kang, S. Jeong, Y. Park, J. Yim, B. Jun, S. Kyeong, J. Yang, G. Kim, S. Hong, L.P. Lee, NearInfrared SERS Nanoprobes with Plasmonic Au/Ag Hollow-Shell Assemblies for In Vivo Multiplex Detection, Adv. Funct. Mater. 23 (2013) 3719-3727.

[20] B. Feng, J. Zhu, B. Lu, F. Liu, L. Zhou, Y. Chen, Achieving infrared detection by All-Si plasmonic hot-electron detectors with high detectivity, ACS Nano. 13 (2019) 8433-8441.

[21] M. Bashirpour, J. Poursafar, M. Kolahdouz, M. Hajari, M. Forouzmehr, M. Neshat, H. Hajihoseini, M. Fathipour, Z. Kolahdouz, G. Zhang, Terahertz radiation enhancement in dipole photoconductive antenna on LT-GaAs using a gold plasmonic nanodisk array, Opt. Laser Technol. 120 (2019) 105726.

[22] N.C. Das, K.K. Choi, Gold plasmonic material for enhanced $\mathrm{Hg} 1-\mathrm{xCdxTe}$ infrared absorption, 
AIP Adv. 9 (2019) 105021.

[23] S. Ghorbani, M. Bashipour, M. Kolahdouz, Improving Unbiased Terahertz Photoconductive Antenna Based on Dissimilar Schottky Barriers Using Plasmonic Mode Excitation, Optik (Stuttg). (2019) 162975.

[24] M. Bashirpour, S. Ghorbani, M. Kolahdouz, M. Neshat, M. Masnadi-Shirazi, H. Aghababa, Significant performance improvement of a terahertz photoconductive antenna using a hybrid structure, RSC Adv. 7 (2017). https://doi.org/10.1039/c7ra11398f.

[25] J. Poursafar, M. Bashirpour, M. Kolahdouz, A.V. Takaloo, M. Masnadi-Shirazi, E. Asl-Soleimani, Ultrathin solar cells with Ag meta-material nanostructure for light absorption enhancement, Sol. Energy. 166 (2018) 98-102.

[26] K. Zhou, Q. Cheng, L. Lu, B. Li, J. Song, Z. Luo, Dual-band tunable narrowband near-infrared light trapping control based on a hybrid grating-based Fabry-Perot structure, Opt. Express. 28 (2020) 1647-1656.

[27] R. Stanley, Plasmonics in the mid-infrared, Nat. Photonics. 6 (2012) 409-411.

[28] S. Adachi, Optical dispersion relations for GaP, GaAs, GaSb, InP, InAs, InSb, Al x Ga1- x As, and In1-x Ga x As y P1-y, J. Appl. Phys. 66 (1989) 6030-6040.

[29] S.A. Maier, Plasmonics - Fundamentals and Applications, Springer, 2007.

[30] P. Karpinski, A. Miniewicz, Surface Plasmon Polariton Excitation in Metallic Layer Via Surface Relief Gratings in Photoactive Polymer Studied by the Finite-Difference Time-Domain Method, Plasmonics. 6 (2011) 541-546. https://doi.org/10.1007/s11468-011-9234-3. 